ORIGINAL ARTICLE

\title{
Is a chest pain observation unit likely to be cost effective at my hospital? Extrapolation of data from a randomised controlled trial
}

\section{S Goodacre, S Dixon}

Emerg Med J 2005;22:418-422. doi: 10.1136/emj.2004.016881

See end of article for authors' affiliations

Correspondence to:

Dr S Goodacre, Medical Care Research Unit, Regent Court, 30 Regent Street, Sheffield, S1 4DA; s.goodacre@sheffield. ac.uk

Accepted for publication 11 May 2004 chest pain observation unit (CPOU) care appeared to be cost Objectives: The ESCAPE trial showed that chest pain observation unit (CPOU) care appeared to be cost
effective compared with routine care. This finding may not be generalisable to hospitals that currently admit fewer patients than the trial hospital or that require higher direct costs to provide CPOU care. This study aimed to explore these issues in sensitivity analyses and develop a nomogram to allow prediction of whether a CPOU will be cost effective in a specific hospital.

Methods: Data from the ESCAPE trial was used to populate a decision analysis model comparing CPOU with routine care. Sensitivity analyses examined the effect of varying the admission rate with routine care and the direct running costs of CPOU care following which the nomogram was created.

Results: CPOU care provided improved outcome $(0.3936$ v 0.3799 QALYs) at lower cost ( $£ 478 v £ 556$ per patient), with fewer patients admitted (37\% v 54\%). Mean cost of CPOU and routine care was $£ 116$ and $£ 73$, respectively, and of inpatient hospital stay was $£ 312$. The mean post-discharge cost for CPOU and routine care was $£ 253$ and $£ 309$, respectively. Sensitivity analyses showed that CPOU care will not reduce costs at a hospital that currently admits fewer than $35 \%$ of patients, or a hospital that expects to incur direct CPOU running costs of $£ 60$ per patient more than the trial hospital.

Conclusions: Findings of the ESCAPE trial are likely to be generalisable to most settings. The nomogram presented here can be used to predict cost effectiveness in a specific hospital.
A chest pain observation unit (CPOU) aims to provide a cost-effective alternative to routine care for patients presenting to the emergency department (ED) with acute, undifferentiated chest pain. ${ }^{1}$ Patients receive up to six hours of observation and biochemical cardiac testing, followed by, if appropriate, an exercise treadmill test. ${ }^{2}$ The ESCAPE (effectiveness and safety of chest pain assessment to prevent emergency admissions) randomised controlled trial of CPOU versus routine care showed that CPOU care was more effective than routine care and was likely to be cost effective in the National Health Service (NHS) in the UK. ${ }^{3}$

Although this large randomised study provides valuable evidence for CPOU cost effectiveness, we must be cautious about extrapolating the results to hospitals throughout the NHS. Differences between hospitals may limit the generalisability of findings. Two factors in particular are likely to vary among hospitals and have a powerful influence on cost effectiveness. Firstly, the proportion of patients admitted to hospital without a CPOU varies from less than $20 \%$ to over $80 \%{ }^{4}$ Since CPOU saves costs by reducing admissions, the potential for CPOU care to be cost effective will be highly dependent on the proportion admitted with routine care. If a hospital without a CPOU currently admits few patients, there is little scope for the CPOU to reduce costs. Secondly, the direct cost of providing CPOU care is an important determinant of cost effectiveness. ${ }^{5}$ This cost is likely to depend upon local factors, such as the availability of appropriately trained staff, short stay facilities, chemical pathology services, and exercise treadmill facilities.

The difficulties of extrapolating findings from one setting to another are well recognised in economic evaluation and modelling techniques have been developed to enhance the transferability of data. ${ }^{6}$ We aimed to use these techniques to undertake a sensitivity analysis of the findings of the ESCAPE trial to determine the potential effect on CPOU cost effectiveness of variation in (a) the proportion of patients currently admitted by a hospital without CPOU care, and $(b)$ the direct cost of providing CPOU care. This will assist clinicians and managers to determine whether establishing CPOU care is likely to be cost effective in their local setting.

\section{METHODS}

The CPOU at the Northern General Hospital in Sheffield is based in the trolley bay area of the ED. It is staffed by three G grade chest pain nurses and is open from 9 am to 9 pm every day. The chest pain nurses are responsible for caring for all patients presenting with definite or suspected acute coronary syndrome, regardless of whether they are cared for on the CPOU. Patients receive continuous ST segment monitoring for two to six hours. ${ }^{8}$ Biochemical cardiac testing consists of creatine kinase (CK)-MB (mass) measurement at presentation and at least two hours later, ${ }^{9}$ and troponin T measurement at least six hours after the worst episode of pain. ${ }^{10}$ Following this an exercise treadmill test is performed using a machine based in the CPOU, unless the patient is unable to exercise or had recent definitive testing for coronary heart disease. Medical input is provided by middle grade ED staff.

The ESCAPE trial was a single centre cluster randomised controlled trial comparing CPOU with routine care at the Northern General Hospital in Sheffield. From 5th February 2001 to 5th May 2002, days of the week were randomised to CPOU or routine care. Patients attending the ED with acute, undifferentiated chest pain were selected and asked to participate. If they consented to participate they were followed up over the following six months. Full details of the CPOU protocol ${ }^{2}$ and the ESCAPE trial $^{3}$ have been published elsewhere.

Abbreviations: $C P O U$, chest pain observation unit; ED, emergency department; QALY, quality adjusted life year 


\section{Cost effectiveness analysis}

The cost effectiveness analysis took a health service costing perspective. Costs relating to the initial attendance with chest pain were identified and measured up to six months after attendance, and valued as Pounds Sterling (2001-02 prices) using a mixture of local and national unit costs. ${ }^{11}{ }^{12}$ We measured resource use at aggregate level for all patients in the trial (that is, the number of days in hospital, outpatient visits, investigations performed etc.). Resource use over the initial six hours of care was measured by direct observation for a subgroup of 96 patients to obtain precise estimates during this period, when CPOU and routine care was likely to be most different. The outcomes were measured using the self-complete EQ-5D questionnaire at two days, one month, and six months, and valued as quality adjusted life years (QALYs) over six months.

Our analysis compared the costs and outcomes of CPOU and routine care. If one or other form of care (CPOU or routine care) provided improved outcomes at a lower cost then it could be said to dominate the alternative and was clearly the preferred option. If, however, one alternative was more effective and more expensive, we estimated the incremental cost per QALY gained of providing that alternative. This is the additional cost required to accrue an additional QALY by providing the more expensive alternative. ${ }^{13}$ The incremental cost per QALY of providing a more effective, more expensive strategy can be compared to the incremental cost per QALY of other healthcare interventions to determine whether it provides value for the additional funding required to support it. ${ }^{14}$

\section{Sensitivity analysis}

We undertook sensitivity analysis by decision analysis modelling, using TreeAge DATA 3.5 software. Decision analysis modelling is a widely used technique that allows estimation of the effect that varying specific variables (in this case the proportion admitted with routine care and the cost of providing $\mathrm{CPOU}$ care) have on our estimate of cost effectiveness.

In the model we assumed that the health service costs comprised three elements:

- ED costs accrued during the initial six hours of care

- costs of hospital admission accrued by patients admitted after the initial six hours of care

- post-discharge costs accrued after hospital discharge, up to six months after attendance.

We assumed that all patients accrued ED and postdischarge costs, and that the value of these costs depended upon whether the patient received CPOU or routine care. We assumed that only admitted patients accrued hospital costs. The average cost of hospital admission for ED or CPOU patients would thus depend upon the probability of admission with CPOU or routine care.

The decision tree outlined in fig 1 was constructed to allow comparison of a decision to manage patients with a CPOU to a decision to use routine care. With each strategy (CPOU or routine care) patients are admitted or discharged according to the probability of admission with CPOU care (padcpou) or the probability of admission with routine care (padroutine). These probabilities are derived from the ESCAPE trial. ${ }^{3}$ Admitted patients accrue costs associated with admission (cADMIT). These costs were estimated from the trial data by calculating the mean costs of inpatient stay for all admitted patients.

The ED costs associated with CPOU and routine care (cEDcpou or cEDroutine) were estimated from the trial data by calculating the mean ED costs for each group. The postdischarge costs associated with $\mathrm{CPOU}$ and routine care (cOTHERcpou or cOTHERroutine) were estimated from the trial data by calculating the mean cost of post-discharge chest pain related care for each group. This included outpatient reviews, diagnostic tests, ED reattendance, hospital readmission, and interventional cardiology procedures. We assumed that outcomes were determined by whether CPOU or routine care was provided and not by whether the patient was admitted to hospital. Outcomes over the initial six months after attendance were measured in the trial.

We addressed the objectives of our study, using the model and trial data, as follows:

- The effect of varying the proportion admitted with routine care was estimated by varying the parameter padroutine from $20 \%$ to $80 \%{ }^{4}$

- The effect of variation in direct CPOU running costs was estimated by varying the estimated ED costs associated with CPOU (cEDcpou) from $£ 20$ less than the baseline estimate to $£ 80$ more than the baseline estimate.

For each potential value of these two parameters the model calculated the estimated cost difference between CPOU and routine care, and the cost effectiveness (incremental cost per QALY) of CPOU compared to routine care. So, for example, the model estimated the cost difference and cost effectiveness of CPOU care if routine care involves admitting 20\% of patients and CPOU costs $£ 20$ per patient less than the baseline estimate. CPOU costs were then incrementally increased (with routine care admission rate fixed) and calculations repeated for each value. This process was then repeated for each potential value of the routine care admission rate.

We then used the results to construct a nomogram that could be used to predict the likely overall cost saving, or additional cost, associated with CPOU care, if the anticipated direct running cost and proportion of patients currently

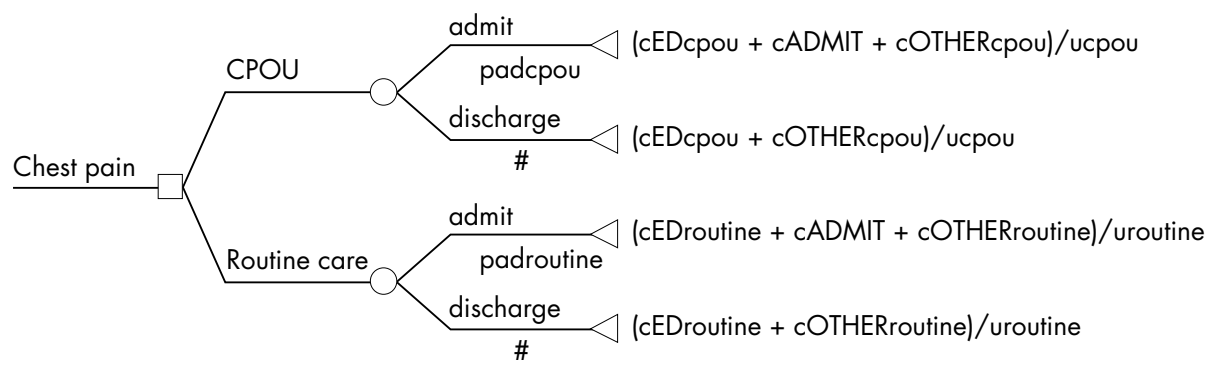

Figure 1 Decision tree used to compare chest pain observation unit (CPOU) and routine care. See Appendix for definitions and values of parameters used in the decision tree. 
admitted were known. Where this predicted that CPOU care was more expensive than routine care, we estimated the incremental cost per QALY.

\section{RESULTS}

The proportion admitted was $36.7 \%$ (176/479) with CPOU and $53.8 \%(265 / 493)$ with routine care. Over the six months following attendance patients receiving CPOU care accrued a mean of 0.3936 QALYs compared with 0.3799 QALYs after routine care (difference 0.0137 QALYs, 95\% CI 0.0030 to $0.0254, \mathrm{p}=0.022$ ). This is equivalent to a $2.7 \%$ improvement in quality of life over six months. The mean cost per patient for chest pain related care over six months was $£ 478$ for the CPOU group and $£ 556$ for the routine care group (difference $£ 78$ per patient, $95 \% \mathrm{CI}-£ 56$ to $£ 210, \mathrm{p}=0.252$ ). The mean $\mathrm{ED}$ costs of CPOU and routine care were $£ 116$ and $£ 73$, respectively. The mean cost of inpatient hospital stay was £312. The mean post-discharge cost associated with CPOU and routine care was $£ 253$ and $£ 309$, respectively.

The estimate of $£ 116$ for the ED costs of CPOU care comprised $£ 15$ staff costs, $£ 31$ overheads, $£ 47$ for diagnostic tests (ST segment monitoring, electrocardiograms, blood tests and $x$ rays), and $£ 23$ for the treadmill test. The unit cost of a CPOU treadmill test was estimated, using micro-costing by direct observation, to be $£ 35$, and $314 / 479$ patients $(66 \%)$ received a CPOU treadmill test.

When the baseline estimates were used, the model produced (as expected) similar results to the trial. CPOU dominated routine care by accruing an additional 0.0137 QALYs per patient with costs that were $£ 66$ per patient lower. Results of the sensitivity analysis are shown in table 1 . The incremental cost of providing CPOU care is shown for each value of the two parameters (negative values indicate that CPOU is cost saving). If CPOU costs more than routine care, then the incremental cost per QALY of providing CPOU care is calculated. If CPOU costs less, it dominates routine care by providing improved outcomes at lower cost.

Figure 2 shows the nomogram for estimating CPOU cost effectiveness. To estimate the likely cost effectiveness of CPOU care at a hospital currently providing routine care one must estimate how the direct cost per patient of providing CPOU care will compare to the baseline of the Northern General Hospital ( $£ 116$ per patient) and estimate the current proportion admitted to hospital. These values are plotted on the first and second bars of the nomogram. A line is drawn connecting these two points and continuing across the third bar. The point where the line crosses the third bar estimates the incremental cost of CPOU care and the incremental cost per QALY of CPOU care, if it is more expensive than routine care.

$\begin{array}{lccc}\text { CPOU cost } & \text { Admission } & \text { Incremental } & \text { Increment cost } \\ \text { per patient } & \text { rates } & \text { NHS cost }(£) & \text { per QALY }(£)\end{array}$

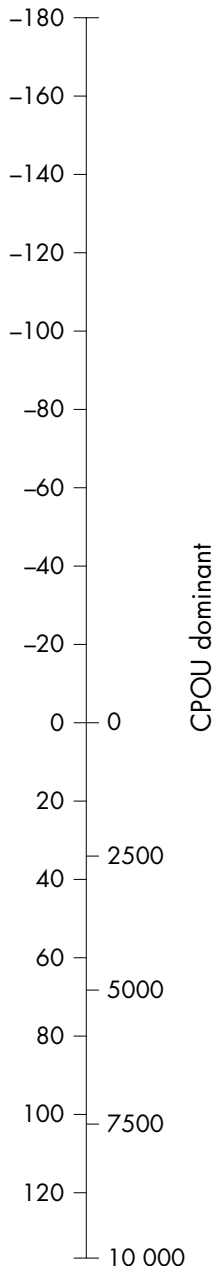

Figure 2 Nomogram to assess chest pain observation unit (CPOU) cost effectiveness for different rates of admission and different direct CPOU costs per patient. NHS, National Health Service; QALY, quality adjusted life year.

\section{DISCUSSION}

The findings of the ESCAPE trial, that CPOU care dominates routine care by providing improved outcomes at lower cost, are only likely to be reproduced elsewhere if:

Table 1 Sensitivity analysis showing the effect of variation in the proportion admitted with routine care and direct cost of providing CPOU care on the difference in cost per patient and cost effectiveness (cost per QALY)

\begin{tabular}{|c|c|c|c|c|c|}
\hline & \multicolumn{5}{|c|}{ Proportion admitted with routine care } \\
\hline & $20 \%$ & $35 \%$ & $50 \%$ & $65 \%$ & $80 \%$ \\
\hline \multicolumn{6}{|c|}{ Direct cost of providing CPOU care } \\
\hline$+£ 80$ & $£ 119$ (£8694 per QALY) & $£ 73$ (£5278 per QALY) & $£ 26$ (£1862 per QALY) & $-£ 21$ & $-£ 68$ \\
\hline$+£ 60$ & £99 (£7234 per QALY) & $£ 53$ (£3818 per QALY) & $£ 6$ (£402 per QALY) & $-£ 41$ & $-£ 88$ \\
\hline$+£ 40$ & $£ 79$ (£5774 per QALY) & $£ 33$ (£2358 per QALY) & $-£ 14$ & $-£ 61$ & $-£ 108$ \\
\hline$+£ 20$ & $£ 59$ (£4314 per QALY) & $£ 13$ (£898 per QALY) & $-£ 34$ & $-£ 81$ & $-£ 128$ \\
\hline Baseline & $£ 39$ (£2854 per QALY) & $-£ 7$ & $-£ 54$ & $-£ 101$ & $-£ 148$ \\
\hline$-£ 20$ & $£ 19$ (£1394 per QALY) & $-£ 27$ & $-£ 74$ & $-£ 121$ & $-£ 168$ \\
\hline
\end{tabular}

Positive values indicate that CPOU costs more-figure in parenthesis is the incremental cost per QALY.

Negative values indicate that CPOU costs less and dominates routine care-no cost per QALY calculated.

CPOU, chest pain observation unit; QALY, quality adjusted life year. 
- current routine practice involves admitting more than 35\% of patients with acute undifferentiated chest pain, and

- the direct costs of providing CPOU care do not exceed those of the Northern General Hospital by more than $£ 60$ per patient.

Even if these conditions are not met and CPOU care is more expensive, it may still be considered cost effective. The incremental cost per QALY of CPOU care, even with high direct costs and compared with a low admission rate with routine care, is relatively modest compared with the threshold value of $£ 30000$ per QALY for new healthcare interventions that seems to guide decision making by the National Institute for Clinical Excellence. ${ }^{14}$ Under these circumstances providing CPOU care will increase health service costs, but these increased costs may be justified by the evidence of improved patient outcomes.

There are a number of reasons why the direct costs of running a CPOU may exceed those estimated at the Northern General Hospital. Firstly, our CPOU is based in an existing area of the ED that did not require extensive redesign or reconstruction. Secondly, our biochemistry laboratories are able to provide troponin and $\mathrm{CK}-\mathrm{MB}$ (mass) results with a rapid turnaround time $(<1$ hour $)$ without additional expense. This would not necessarily be the case if a CPOU required point-of-care tests. Thirdly, treadmill testing is provided at relatively low cost by chest pain nurses within the ED. Finally, we assumed that the chest pain nurses are usefully employed in the ED when not caring for CPOU patients. This explains the relatively low staff costs. If, however, this were not the case then the true cost of providing CPOU care would be higher. In all these cases the nomogram allows clinicians and managers to estimate the influence of additional costs upon the overall cost effectiveness of CPOU care.

In developing the nomogram we have assumed that the admission rate associated with CPOU care is constant at $36.7 \%$. Thus, in some circumstances, the CPOU is both more costly and more likely to result in an admission. In these situations CPOU cost effectiveness is generated by the cost savings and QALY gains after discharge. This assumption of CPOU and routine admission rates being independent is conservative (that is, likely to discriminate against CPOU cost effectiveness). Hospitals that currently have relatively low admission rates with routine care might also be expected to have low admissions rates with CPOU care.

Two further clinical realities are not incorporated within the modelling presented here. Firstly, it is likely that the incidence of acute coronary syndrome varies among patient populations. This could influence the difference in admission rates between routine and CPOU care, and therefore impact on cost effectiveness. The precise effect of this is difficult to estimate, as the current admission rate is also likely to be related to the incidence of acute coronary syndrome. Secondly, the cost and QALY consequences of routine treatment may depend upon admission rate. Lower admission rates are likely to be related to a higher risk of discharge with acute coronary syndrome. ${ }^{15}$ This would reduce quality of life and increase costs after discharge, both of which would improve the cost effectiveness of CPOU. Consequently, the cost increases associated with implementing a CPOU in low routine admission setting are likely to underestimate its clinical and economic benefits.

This study has limitations that should be appreciated. The limitations of the ESCAPE trial (inability to conceal the allocation schedule or blind participants to the intervention) have been discussed elsewhere. ${ }^{3}$ The principal limitation of this analysis is that modelling can only tell us what ought to happen, given the assumptions upon which the model is based. It cannot tell us what will happen. The only sure way to estimate the costs of providing CPOU in a specific hospital is to set up a CPOU and measure the costs. Establishment of a CPOU involves reorganisation of hospital services. The costs and effects of such a change will be highly dependent upon organisational factors such as departmental structure, staffing, case mix and funding. Further research therefore needs to focus upon the organisation factors that influence development of CPOU care in various different settings.

Also, when assessing the implementation of these findings some thought needs to be given to the precise meaning of "cost saving" within economic evaluation. If "cost saving" refers to resources that can be withdrawn (such as additional diagnostic tests) then savings will be generated. However, if it refers to resources that may be difficult to withdraw, such as hospital beds, then these resources are in effect immediately "reinvested" in the treatment of other patients. This may not be the most efficient alternative use for these resources.

A number of economic analyses of CPOU care have been undertaken alongside randomised trials in the USA. ${ }^{16-18}$ However, these trials compared CPOU care with inpatient hospital care. The ESCAPE trial is, to our knowledge, the only randomised trial to compare CPOU with a pragmatic alternative including both hospital admission and discharge. Hence the importance of being able to extrapolate these data to other settings.

Economic analyses may be criticised for producing results that are difficult for clinicians and managers to understand or apply to their local circumstances. In this study we have attempted to address this criticism by using a novel approach to the presentation of the results of sensitivity analysis. We hope that the nomogram will provide a simple way of extrapolating the results on an economic evaluation in one setting to other hospitals in the NHS.

\section{ACKNOWLEDGEMENTS}

We thank all the members of the ESCAPE Research Team: J Nicholl, F Morris, S Capewell, L Cross, J Arnold, K Angelini, S Revill, T Locker, D Quinney, and S Campbell.

\section{Authors' affiliations}

S Goodacre, Medical Care Research Unit, University of Sheffield, Sheffield, UK

S Dixon, Sheffield Health Economics Group, University of Sheffield, Sheffield, UK

Competing interests: none declared

\section{APPENDIX}

Definitions and values of parameters used in the decision tree

\begin{tabular}{lll}
\hline Parameter & Abbreviation & $\begin{array}{l}\text { Baseline } \\
\text { estimate }\end{array}$ \\
\hline Probability of admission with CPOU care & $\begin{array}{l}\text { padcpou } \\
\text { Probability of admission with routine care }\end{array}$ & $\begin{array}{l}\text { padroutine } \\
\text { ucpou }\end{array}$ \\
Utility after CPOU care & $53.8 \%$ \\
Utility after routine care & uroutine & 0.3936 \\
ED costs with CPOU care & cEDcpou & $£ 116$ \\
ED costs with routine care & cEDroutine & $£ 73$ \\
Other costs with CPOU care & cOTHERcpou & $£ 252$ \\
Other costs with routine care & cOTHERroutine & $£ 308$ \\
Cost of hospital admission & cADMIT & $£ 312$ \\
\hline & & \\
\hline
\end{tabular}




\section{REFERENCES}

1 Clancy M. Chest pain units. BMJ 2002;325:116-17.

2 Goodacre S, Morris FP, Campbell S, et al. A prospective, observational study and cost analysis of a chest pain observation unit. Emerg Med J 2002;19:117-121.

3 Goodacre S, Nicholl J, Dixon S, et al. Randomised controlled trial and economic evaluation of a chest pain observation unit versus routine care. BMJ 2004;328:254-7.

4 Goodacre S, Nicholl J, Beahan J, et al. National survey of emergency department management of patients with acute, undifferentiated chest pain. B J Cardiol 2003; 10:50-4.

5 Goodacre S, Calvert N. Cost-effectiveness of diagnostic strategies for acute undifferentiated chest pain. Emerg Med J 2003;20:429-33.

6 Buxton MJ, Drummond MF, Van Hout BA, et al. Modelling in economic evaluation: an unavoidable fact of life. Health Econ 1997;6:217-27.

7 Drummond MF, Pang F. Transferability of economic evaluation results. In: Drummond MF, McGuire A, eds. Economic evaluation in health care. New York: Oxford University Press, 2001:256-76

8 Fesmire FM, Percy RF, Bardoner JB, et al. Usefulness of automated serial 12 lead ECG monitoring during the initial emergency department evaluation of patients with chest pain. Ann Emerg Med 1998;31:3-11.

9 Fesmire FM, Percy RF, Bardoner JB, et al. Serial creatinine kinase (CK) MB testing during the emergency department evaluation of chest pain: utility of a 2-hour deltaCK-MB of +1.6 ng/ml. Am Heart J 1998;136:237-44.
10 Hamm CW Goldman BW, Heeschen C, et al. Emergency room triage of patients with acute chest pain by means of rapid testing for cardiac troponin $\mathrm{T}$ or troponin I. N Engl J Med 1997;337:1648-53

11 NHS Department of Health. National Schedule of Reference Costs. www.doh.gov.uk/nhsexec/refcosts.htm, 2002 (accessed 5 January 2004).

12 Netten A, Rees T, Harrison G. Unit costs of health and social care. Persona Social Services Research Unit, University of Kent, 2001

13 Drummond MF, O'Brien B, Stoddart GL, et al. Methods for the economic evaluation of health care programmes. New York: Oxford University Press, 1997.

14 Raftery J. NICE: faster access to modern treatments? Analysis of guidance on health technologies. BMJ 2001;323:1300-3

15 Graff LG, Dallara J, Ross MA, et al. Impact on the care of the emergency department chest pain patient from the chest pain evaluation registry (CHEPER) study. Am J Cardiol 1997;80:563-8.

16 Farkouh ME, Smars PA, Reeder GS, et al. A clinical trial of a chest pain observation unit for patients with unstable angina. N Engl J Med 1998;339:1882-8

17 Roberts RR, Zalenski RJ, Mensah EK, et al. Costs of an emergency departmentbased accelerated diagnostic protocol vs hospitalization in patients with chest pain. A randomized controlled trial. JAMA 1997;278:1670-6.

18 Gomez MA, Anderson JL, Karagounis LA, et al. An emergency department based protocol for rapidly ruling out myocardial ischaemia reduces hospital time and expense: results of a randomized study (ROMIO). J Am Coll Cardiol 1996;28:25-33.

\section{Call for papers}

11 th European Forum on Quality Improvement in Health Care 26-28 April 2006, Prague, Czech Republic

Deadline 30 September 2005.

For further information and to submit online go to: www.quality.bmipg.com 